\title{
The good, bad, and ugly of incidental findings on cardiovascular-computed tomography
}

\author{
Pradeep Bhambhvani, $M^{a}$ \\ ${ }^{a}$ Division of Molecular Imaging and Therapeutics, Department of Radiology, The University of \\ Alabama at Birmingham, Birmingham, AL
}

Received Jul 9, 2015; accepted Jul 10, 2015

doi: $10.1007 / \mathrm{s} 12350-015-0244-y$

\section{See related article, pp.1266-1274}

Technological advances have led to increased utilization of computed tomography (CT) for cardiovascular imaging. Common indications include coronary calcium assessment, detection of obstructive coronary artery disease (CAD) with coronary CT angiography (CCTA), and for attenuation correction (CTAC) when using myocardial perfusion imaging with single-photon emissioncomputed tomography (SPECT) or positron emission tomography (PET). ${ }^{1}$ CTAC is known to improve diagnostic accuracy by reducing attenuation artifact and has been shown to improve the specificity of SPECT and PET in the detection of obstructive CAD. ${ }^{1}$

In addition to the heart, the imaging field of view of cardiovascular CT usually includes portions of the lungs, pleura, mediastinum, chest wall, bones, upper abdomen, and the thoracic aorta. The entire thorax is usually never imaged during normal acquisition as the superior and inferior borders of the heart serve as landmarks to the image. The usual small field of view for cardiac CT and the restricted superior and inferior borders allow only $\sim 10 \%$ of lung tissue to be visualized. Thus, a lung study derived from a cardiac CT study will always be incomplete and will exclude at least the apices and possibly the bases. ${ }^{2}$ Limiting the number of slices by reducing the length along the $\mathrm{z}$-axis is done to reduce the patient radiation exposure. ${ }^{2}$

Reprint requests: Pradeep Bhambhvani, MD, Division of Molecular Imaging and Therapeutics, Department of Radiology, The University of Alabama at Birmingham, Birmingham, AL; pbhambhvani@ uabmc.edu

J Nucl Cardiol 2016;23:1275-9.

$1071-3581 / \$ 34.00$

Copyright (C) 2015 American Society of Nuclear Cardiology.
The benefits of cardiovascular CT including the highspatial and temporal resolution and attenuation correction are accompanied with risks of radiation exposure and the unintended consequences of extracardiac incidental findings on patient quality of life, outcomes, and costs related to downstream resource utilization.

An incidental finding (IF) may be defined as an incidentally discovered mass or lesion, detected by an imaging examination performed for an unrelated reason. ${ }^{3}$ Common extracardiac IFs seen with cardiac CT include pulmonary nodules, emphysema, bronchiectasis, pleural effusion, diaphragmatic hernia, liver nodule/cyst, liver steatosis, aortic dilation, other vascular anomalies, adenopathy, and many others. ${ }^{4}$ The prevalence of noncardiac IFs ranges from $8 \%$ to $69 \%$ depending on the type of CT protocol used and age of the participants with the most frequent IFs being pulmonary nodules. ${ }^{5}$ IFs can be classified as benign, i.e., of no clinical importance and requiring no additional work-up or follow-up or a clinically significant finding, which is a potentially or definitely important lesion requiring additional investigation. This second category can be subdivided into indeterminate findings requiring clinical correlation or follow-up and major findings requiring immediate evaluation or management. Approximately $4 \%$ to $25 \%$ of findings are reported to be potentially significant, and $5 \%$ to $11 \%$ are reported to be major, requiring immediate evaluation or intervention. ${ }^{6}$ Interestingly, IFs are reportedly found more frequently than the diagnostic entities for which studies are primarily targeted. ${ }^{7}$ A study of CT angiography for pulmonary embolism, reported incidental findings requiring follow-up were nearly 3 times more common than emboli. ${ }^{8}$

Although most IFs prove to be benign, their discovery often leads to a cascade of testing that is costly, and exposes patients to unnecessary additional radiation. ${ }^{9}$ The most common reason to pursue incidental findings is to differentiate benign from potentially serious (including malignant) lesions. ${ }^{9}$ Additional imaging is 
often the next step in the evaluation of an unexpected IF. Other interventions include primary care office visits, subspecialist referrals, diagnostic and therapeutic procedures, surgical procedures, and hospitalizations related to these procedures with considerable costs involved. ${ }^{10}$ Although there are several publications in the literature addressing costs associated with IFs. ${ }^{4,10}$ The reverse calculations estimating health care cost savings related to future morbidity and mortality had these findings gone undiscovered, cannot be quantified and remain unclear. IF-related costs have also raised concerns with the government and third-party payers as medical imaging utilization have risen. For example, the noncoverage decision for screening computed tomography colonography for colorectal cancer by CMS noted concern for the costs of evaluating extracolonic findings that are diagnostically indeterminate. ${ }^{11}$

A recently published study concluded that routine follow-up of incidentally detected pulmonary nodules in stable symptomatic patients undergoing CCTA resulted in a small reduction in lung cancer mortality ( $8 \%$ at 3 years) at the cost of significant downstream testing, leading to a high cost per quality-adjusted life-year $(\$ 154,700)$, especially in nonsmokers. Follow-up strategy was less expensive and more efficient in smokers $(\$ 129,800)$ per quality-adjusted life-year. ${ }^{12}$ The authors argue that this cost is greater than the generally accepted threshold (\$100,000 per quality-adjusted life-year) for approval or payment of medical interventions. The concept of cost effectiveness although pragmatic and a must discussion in the era of ever increasing health care expenses is also very challenging and nebulous to say the least. This concept has been very well characterized by an apt remark "Cost effectiveness in medicine often depends on whose life is being saved, mine or somebody else's', 13

In addition to costs, downstream testing and interventions for IFs are also likely to provoke anxiety in patients and their family members and may cause morbidity from adverse effects of the tests or treatments. ${ }^{14}$

Other related challenges include what findings to report, how to report, and how best to follow-up with the physician or patient on these findings. There exists guidance from the Fleischner Society position papers on management of subsolid and solid pulmonary nodules detected at CT and pulmonary embolism in the era of CT angiography. ${ }^{15-17}$ However, the significance and management of various other extracardiac IFs remain unclear and indeterminate. Also even when the guidelines exist, reporting practices are variable. Several radiologists conform to the guidelines, but many do not. When there are no guidelines, the variability is likely even greater. $7,18,19$ Comparison with prior imaging when available, to establish stability and/or resolution vs growth is an elementary technique very useful in characterizing lesion/disease biology and prudent resource utilization. According to the American College of Radiology practice parameter for communication of diagnostic imaging findings, comparison with relevant examinations and reports should be part of the radiologic consultation and report when appropriate and available. ${ }^{20}$ In the context of 2-year stability of pulmonary nodules, Yankelevitz and Henschke concluded that lack of growth implies a long doubling time, which generally yields a benign histology. Even in those cases that are malignant, the lung cancers with longer doubling times tend to have a better prognosis. ${ }^{21}$

Depending on the clinical indication, three types of cardiovascular CT protocols have been described: an unenhanced, nongated, free tidal breathing CT scan (5-mm slice thickness) for attenuation correction; an unenhanced, gated, breath-hold CT scan (2- to 3-mm slice thickness) for coronary artery calcium scoring; and a contrast-enhanced, gated, breath-hold CT scan (0.5- to 0.75-mm slice thickness) for coronary CTA. ${ }^{1}$ The above protocols differ not only in technique but are also notable for increasing ionizing radiation exposure. ${ }^{22}$ Collaborative guideline by the Society of Nuclear Medicine and Molecular Imaging, American Society of Nuclear Cardiology and Society of Cardiovascular Computed Tomography recommends CT scanning for attenuation correction with shallow tidal breathing, tube current and voltage of approximately 10$20 \mathrm{~mA}$ and $80-140 \mathrm{kVp}$, gantry rotation speed of $1 \mathrm{~s}$ or slower, relatively high pitch (e.g., 1:1), and reconstructed slice thickness of 4-7 mm approximating SPECT or PET. ${ }^{1}$ In comparing standard-dose (120 kVp, 40-100 mA) vs low-dose (120 kVp, 20-60 mA) CTAC on image quality and PET attenuation correction, Krishnasetty et al have demonstrated that the low-dose CTAC significantly reduced the radiation effective dose (by 60.6\%) while providing optimal PET attenuation correction. However, because of decreased image quality in both anatomic definition and noise, low-dose CTAC was not acceptable for diagnostic interpretation. ${ }^{23}$ Compared to standard-dose $\mathrm{CT}$, the inferior image quality of the low-dose CTAC as shown in Figure 1 is probably related to a combination of lower tube current-time product expressed in units of milliampere seconds (mAs), free tidal breathing and related artifacts, and lack of administered contrast. An example demonstrating the differences in image quality between low-dose CTAC vs noncontrast-enhanced standard-dose CT is shown in Figure 1.

For diagnostic radiologists, evaluation of all tissues in the X-ray path has long been the standard of care. ${ }^{24}$ Cardiologists interpreting cardiovascular CT examinations may not have the requisite training and/or experience to report extracardiac findings. Available strategies to deal with extracardiac tissues include acquiring additional training to allow confident handling of such findings or separate evaluation by a radiologist for interpretation of 

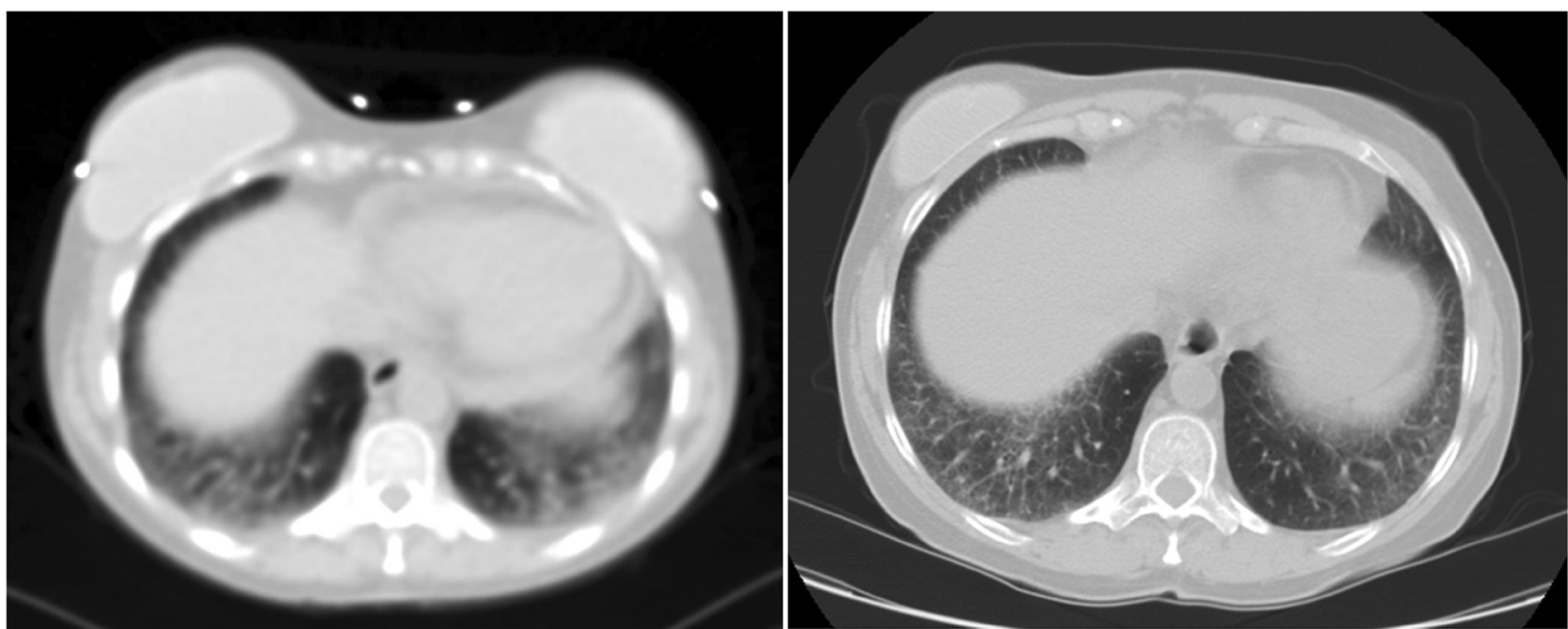

Low Dose CTAC

$\begin{array}{ll}\mathrm{KVp} & 120 \\ \mathrm{~mA} & 20 \\ \text { Gantry Rotation } & 0.8 \mathrm{sec} \\ \text { Breathing } & \text { Free } \\ \text { Slice Thickness } & 5 \mathrm{~mm} \\ \text { Pitch } & 0.938\end{array}$

\begin{tabular}{c} 
Standard Dose CT \\
\hline 120 \\
150 \\
$0.8 \mathrm{sec}$ \\
Hold \\
$5 \mathrm{~mm}$ \\
0.98
\end{tabular}

Figure 1. Note the haziness/blurring in the bilateral lung bases from the inferior image quality of the low-dose CTAC when compared to standard-dose CT.

extracardiac tissues. The latter approach requires additional time and effort that may not be fully reimbursed. Also some practices may not have radiology expertise available. ${ }^{24}$ Another strategy of digital subtraction of extracardiac tissues is mired not only with medical and ethical concerns but is also technically challenging as some lung tissue invariably remains in the field of view. Onuma et al showed that a number of clinically significant noncardiac findings might have been missed with the small field of view (tightly confined around the heart) cardiac multi-detector CT scans. Of the 201 symptomatic patients in whom CAD was ruled out, 32 patients were diagnosed with noncardiac findings on large field of view images that included the entire chest, which could cause anginal symptoms in addition to the 3 cases of malignancies in surgically treatable stages. ${ }^{25}$ A group of South Korean investigators also demonstrate fewer patients with incidental noncoronary findings $(1.6 \%)$ with the small field of view CCTA as compared to $20.4 \%$ of patients with extracardiac IFs with a bigger field of view low-dose whole thoracic scan. ${ }^{26}$

Given the needed knowledge of anatomy, the complexity of imaging devices, protocols, and evolving clinical applications of this modality the American College of Cardiology (ACC) has proposed training and competency requirements for physicians participating in cardiovascular CT which includes identification of significant noncardiac imaging findings. The ACC with representation and endorsement from the American Society of Nuclear Cardiology (ASNC), Society for Cardiovascular Angiography and Interventions (SCAI), Society of Atherosclerosis Imaging and Prevention (SAIP), and Society of Cardiovascular Computed Tomography (SCCT) has published training requirements for fellows-in-training and physicians in practice in Cardiovascular CT. ${ }^{27,28}$ The American College of Radiology also has practice guideline for the performance and interpretation of cardiac CT. ${ }^{29}$

In the current issue of the journal, Qureshi et al discuss the prevalence, prognosis, and resource utilization of extracardiac IFs on CTAC SPECT myocardial perfusion imaging. ${ }^{30}$ They note that IFs are common (12\% prevalence in their cohort) and were associated with higher all-cause and cancer-specific mortality in univariate analysis but only the latter remained statistically significant after multivariable adjustment. Another study involving CCTA did not identify extracardiac IFs as a predictor of noncardiac or cancer death. ${ }^{4}$ In the study by Qureshi et al the observed higher cancerspecific mortality may be due to patient selection, as almost $1 / 3$ of their study population was African American. ${ }^{30}$ An additional reason for the differences in IFs prevalence (12\% vs $41.5 \%$ ) and mortality outcomes may be related to the differences in CT parameters and resulting image quality between studies. The parameters 
of the $120 \mathrm{kVp}, 20-40 \mathrm{~mA}$, free breathing, and noncontrast-enhanced CTAC used by Qureshi et $\mathrm{al}^{30}$ are quite different from the $120 \mathrm{kVp}, 400-800 \mathrm{~mA}$, gantry rotation time of $350 \mathrm{~ms}$, individualized pitch, breath hold, and contrast-enhanced CCTA described by MacHaalany et al. ${ }^{4}$ As noted previously, the low-dose CTAC images are not of diagnostic quality when compared to standard-dose CT. ${ }^{23}$ Simply put the limitations from the lower image quality CTAC would be able to identify the more obvious, larger and advanced extracardiac tumors/disease findings and potentially miss the smaller, earlier stage and possibly innocuous findings resulting in the lower prevalence and higher cancerspecific mortality as observed by Qureshi et al. ${ }^{30}$ Additional studies are needed to confirm their observations and further understand the prognostic implications of the identification of extracardiac IFs.

In summary, with all the uncertainties of incidental findings, the challenge we face as imaging physicians is to balance on one hand the good that a majority of them are benign with the bads of their prevalence, downstream interventions, related costs, patient anxiety, morbidity, potential diagnosis of malignancy, and the ugly possibility of poor patient outcomes (i.e., higher cancerspecific mortality as observed by Qureshi et $\mathrm{al}^{30}$ ) when making recommendations for further testing or treatment.

Our overriding responsibility is always to do right by our patients and profession. This includes but is not limited to keeping up to date with existing and evolving scientific information, proficiency with current and new technologies, comparison with prior imaging to establish stability, and hence probable benignity and/or better prognosis, educating our referral colleagues on appropriate use testing, reporting 'all' findings (incidental and nonincidental) seen in the image, and using evidencebased recommendations where available in our reporting to assist not only the referring physician, but ultimately the patient.

\section{References}

1. Dorbala S, Di Carli MF, Delbeke D, Abbara S, DePuey EG, Dilsizian V, et al. SNMMI/ASNC/SCCT guideline for cardiac SPECT/CT and PET/CT 1.0. J Nucl Med 2013;54:1485-507.

2. Budoff MJ, Fischer H, Gopal A. Incidental findings with cardiac CT evaluation: Should we read beyond the heart? Catheter Cardiovasc Interv 2006;68:965-73.

3. Berland LL. Overview of white papers of the ACR incidental findings committee ii on adnexal, vascular, splenic, nodal, gallbladder, and biliary findings. J Am Coll Radiol 2013;10:672-4.

4. Machaalany J, Yam Y, Ruddy TD, Abraham A, Chen L, Beanlands RS, et al. Potential clinical and economic consequences of noncardiac incidental findings on cardiac computed tomography. J Am Coll Cardiol 2009;54:1533-41.
5. Jaar BG, Zhang L, Chembrovich SV, Sozio SM, Shafi T, Scialla $\mathrm{JJ}$, et al. Incidental findings on cardiac computed tomography in incident hemodialysis patients: the predictors of arrhythmic and cardiovascular events in end-stage renal disease (PACE) study. BMC Nephrol 2014;15:68.

6. Mark DB, Berman DS, Budoff MJ, Carr JJ, Gerber TC, Hecht HS, et al. ACCF/ACR/AHA/NASCI/SAIP/SCAI/SCCT 2010 expert consensus document on coronary computed tomographic angiography: A report of the American College of Cardiology Foundation Task Force on Expert Consensus Documents. Circulation 2010;121:2509-43

7. Brown SD. Professional norms regarding how radiologists handle incidental findings. J Am Coll Radiol 2013;10:253-7.

8. Hall WB, Truitt SG, Scheunemann LP, Shah SA, Rivera MP, Parker LA, et al. The prevalence of clinically relevant incidental findings on chest computed tomographic angiograms ordered to diagnose pulmonary embolism. Arch Intern Med 2009;169:1961-5.

9. Berland LL, Silverman SG, Gore RM, Mayo-Smith WW, Megibow AJ, Yee J, et al. Managing incidental findings on abdominal CT: White paper of the ACR incidental findings committee. J Am Coll Radiol 2010;7:754-73.

10. Morgan AE, Berland LL, Ananyev SS, Lockhart ME, Kolettis PN. Extraurinary incidental findings on CT for hematuria: The radiologist's role and downstream cost analysis. AJR Am J Roentgenol 2015;204:1160-7.

11. Decision Memo for Screening Computed Tomography Colonography (CTC) for Colorectal Cancer (CAG-00396N). May 12, 2009.

12. Goehler A, McMahon PM, Lumish HS, Wu CC, Munshi V, Gilmore $\mathrm{M}$, et al. Cost-effectiveness of follow-up of pulmonary nodules incidentally detected on cardiac computed tomographic angiography in patients with suspected coronary artery disease. Circulation 2014;130:668-75.

13. Rumberger JA. Noncardiac abnormalities in diagnostic cardiac computed tomography: Within normal limits or we never looked! J Am Coll Cardiol 2006;48:407-8.

14. Casarella WJ. A patient's viewpoint on a current controversy. Radiology 2002;224:927.

15. Naidich DP, Bankier AA, MacMahon H, Schaefer-Prokop CM, Pistolesi M, Goo JM, et al. Recommendations for the management of subsolid pulmonary nodules detected at CT: A statement from the Fleischner Society. Radiology 2013;266:304-17.

16. MacMahon H, Austin JH, Gamsu G, Herold CJ, Jett JR, Naidich DP, et al. Guidelines for management of small pulmonary nodules detected on CT scans: A statement from the Fleischner Society. Radiology 2005;237:395-400.

17. Remy-Jardin M, Pistolesi M, Goodman LR, Gefter WB, Gottschalk A, Mayo JR, et al. Management of suspected acute pulmonary embolism in the era of CT angiography: A statement from the Fleischner Society. Radiology 2007;245:315-29.

18. Johnson PT, Horton KM, Megibow AJ, Jeffrey RB, Fishman EK. Common incidental findings on MDCT: Survey of radiologist recommendations for patient management. J Am Coll Radiol 2011;8:762-7.

19. Eisenberg RL, Bankier AA, Boiselle PM. Compliance with Fleischner Society guidelines for management of small lung nodules: A survey of 834 radiologists. Radiology 2010;255:21824.

20. ACR. ACR practice parameter for communication of diagnostic imaging findings 2014.

21. Yankelevitz DF, Henschke CI. Does 2-year stability imply that pulmonary nodules are benign? AJR Am J Roentgenol 1997; 168:325-8. 
22. Hunold P, Vogt FM, Schmermund A, Debatin JF, Kerkhoff G, Budde T, et al. Radiation exposure during cardiac CT: Effective doses at multi-detector row CT and electron-beam CT. Radiology 2003;226:145-52.

23. Krishnasetty V, Bonab AA, Fischman AJ, Halpern EF, Aquino SL. Comparison of standard-dose vs low-dose attenuation correction $\mathrm{CT}$ on image quality and positron emission tomographic attenuation correction. J Am Coll Radiol 2008;5:579-84.

24. Bluemke DA. Coronary computed tomographic angiography and incidental pulmonary nodules. Circulation 2014;130:634-7.

25. Onuma Y, Tanabe K, Nakazawa G, Aoki J, Nakajima H, Ibukuro $\mathrm{K}$, et al. Noncardiac findings in cardiac imaging with multidetector computed tomography. J Am Coll Cardiol 2006;48:402-6.

26. Kim JW, Kang EY, Yong HS, Kim YK, Woo OH, Oh YW, et al. Incidental extracardiac findings at cardiac CT angiography: Comparison of prevalence and clinical significance between precontrast low-dose whole thoracic scan and postcontrast retrospective ECGgated cardiac scan. Int J Cardiovasc Imaging 2009;25:75-81.
27. Garcia MJ, Blankstein R, Budoff MJ, Dent JM, Drachman DE, Lesser JR, et al. COCATS 4 Task Force 7: Training in cardiovascular computed tomographic imaging. J Am Coll Cardiol 2015;65:1810-21

28. Budoff MJ, Cohen MC, Garcia MJ, Hodgson JM, Hundley WG, Lima JA, et al. ACCF/AHA clinical competence statement on cardiac imaging with computed tomography and magnetic resonance: A report of the American College of Cardiology Foundation/American Heart Association/American College of Physicians Task Force on Clinical Competence and Training. J Am Coll Cardiol 2005;46:383-402.

29. Jacobs JE, Boxt LM, Desjardins B, Fishman EK, Larson PA, Schoepf J. ACR practice guideline for the performance and interpretation of cardiac computed tomography (CT). J Am Coll Radiol 2006;3:677-85.

30. Qureshi WT, Alirhayim Z, Khalid F, Al-Mallah MH. Prognostic value of extracardiac incidental findings on attenuation correction cardiac computed tomography. J Nucl Cardiol 2015. doi:10.1007/ s12350-015-0223-3. 